\title{
Effects of Fe substitution on the transport properties of the superconductor $\mathrm{MgB}_{2}$
}

\author{
Bhasker Gahtori, Ratan Lal, and S. K. Agarwal \\ Superconductivity and Cryogenics Division, National Physical Laboratory, Dr. K. S. Krishnan Road, New Delhi 110012, India
}

Y. K. Kuo,* K. M. Sivakumar, and J. K. Hsu

Department of Physics, National Dong Hwa University, Hualien 974, Taiwan

J. Y. Lin

Institute of Physics, National Chiao Tung University, Hsinchu 30050, Taiwan

Ashok Rao

Department of Physics, Manipal Institute of Technology, Manipal 576104, Karnataka, India

S. K. Chen and J. L. MacManus-Driscoll

Department of Materials Science and Metallurgy, University of Cambridge, Pembroke Street, Cambridge CB2 3QZ, United Kingdom

(Received 3 August 2006; revised manuscript received 13 February 2007; published 16 May 2007)

\begin{abstract}
A systematic study has been made on the effect of Fe substitution by means of resistivity, thermal conductivity, and Seebeck coefficient of the $\mathrm{Mg}_{1-x} \mathrm{Fe}_{x} \mathrm{~B}_{2}$ superconductor involving 0\%, 0.3\%, 0.6\%, $1.2 \%$, and 3.0\% Fe content. The superconducting transition has been found to be very sharp $(\sim 0.2 \mathrm{~K})$ for a pristine sample and substitution of $\mathrm{Fe}$ results in the decrease of $T_{C}$ with the increase in the transition width. Thermal conductivity is found to decrease with Fe content in general, such that the shoulder present in the pristine sample tends to fade away with increasing Fe. An analysis has been made on the normal state resistivity in terms of a two-band model, and of the thermal conductivity in terms of the Wiedemann-Franz law and the lattice thermal conductivity, and the information obtained on the basis of this analysis has been discussed. Besides, the electronic density of states (DOS) near the Fermi level remains nearly unaffected upon Fe substitution, as evidenced by the Seebeck coefficient measurements. When compared with Mn, Fe behaves like a nonmagnetic element with a modest variation in $T_{C}$ and on the other hand, the $T_{C}$ depression is much stronger when compared with other elements like $\mathrm{Al}, \mathrm{Cu}$, etc. Therefore, the observed variation in $T_{C}$ for the presently investigated concentrations of $\mathrm{Fe}$ is attributed to the specific nature of the given substituent element $(\mathrm{Fe})$ in altering the phonon frequency and/or electron-phonon coupling strength rather than spin-flip scattering or change in DOS or disorder.
\end{abstract}

DOI: $10.1103 /$ PhysRevB.75.184513

PACS number(s): 74.70.Ad, 74.25.Fy, 74.62.Dh

\section{INTRODUCTION}

Recently reported superconductivity in magnesium diboride $\left(\mathrm{MgB}_{2}\right)$ at $\sim 40 \mathrm{~K}$, highest for an intermetallic compound, ${ }^{1}$ has resulted in intensive investigations both theoretically and experimentally to exploit the possible superconductivity at higher than $40 \mathrm{~K}$ for practical applications, and to understand the underlying mechanisms of the normal and superconducting states of this system. ${ }^{2-5}$ The structure of $\mathrm{MgB}_{2}$ is a simple hexagonal $\mathrm{AlB}_{2}$ type with honeycomb-type boron layers and interpenetrating $\mathrm{Mg}$ layers. ${ }^{3}$ It has been well established that it is a multiband, namely $\sigma$ and $\pi$, and conventional $s$-wave phonon mediated BCS-type superconductor. ${ }^{3}$ The two bands and the respective superconducting gaps are associated with different parts of its Fermi surface. ${ }^{4}$ It is believed that the $\sigma$-band is of hole type and is mainly responsible for the superconductivity in $\mathrm{MgB}_{2}$ whereas the $\pi$-band is an electron type with comparatively negligible contribution. ${ }^{4}$ In spite of the fact that $\mathrm{MgB}_{2}$ is one of the simplest binary compounds with a simple crystal structure, some of its physical, chemical, and electrical properties are very intriguing and not yet fully understood even after numerous investigations. ${ }^{5}$

Chemical substitution, like in other superconductors, serves as a useful tool to modify the structure and other physical properties of $\mathrm{MgB}_{2}$ to study the underlying mechanism of superconductivity and improve some of its important parameters for practical applications. To explain the variations observed in $T_{C}$ upon different substitutions, so far various mechanisms, such as pair breaking effect, reduction in density of states (DOS), decrease in phonon frequency, and band narrowing or expansion due to increase and/or decrease in the lattice constants and/or disorder have been proposed. ${ }^{6-10}$ For example, the reduction in $T_{C}$ with $\mathrm{Al}$ substitution for B has been attributed to the decrease in DOS at $E_{F}$ due to the electron doping in the $\sigma$-band. ${ }^{11,12}$ Moreover, a sharp drop in $T_{C}$ has been reported for the substitution of magnetic element such as Mn, while a different mechanism, spin-flip scattering and/or pair breaking effect has been proposed to the observed $T_{C}$ variation. ${ }^{13,14}$ On the other hand, another magnetic element $\mathrm{Fe}$ had shown much slower variations in $T_{C}$ in comparison with Mn (Refs. 8, 15, and 16) and such a diverse variation between $\mathrm{Fe}$ and $\mathrm{Mn}$ has been attributed to the nonmagnetic nature of $\mathrm{Fe}$ in $\mathrm{Mg}$ lattice. ${ }^{17}$ The major setback with such substitutional studies is the limitation in the solubility of the third element at the $\mathrm{Mg}$ and/or at the $\mathrm{B}$ site, ${ }^{18}$ except for aluminum (Al) and carbon (C), ${ }^{11,19}$ which can be readily substituted at $\mathrm{Mg}$ and $\mathrm{B}$ sites, respectively. Nevertheless, other elements show successful substi- 
tution at $\mathrm{Mg} / \mathrm{B}$ sites only if their concentration is below $5 \%$. $^{6,7,15}$

Among the transition metal substitutes attempted, ${ }^{6-8,10,20-22} \mathrm{Fe}$ substitution in $\mathrm{MgB}_{2}$ behaves in a unique way and is of particular importance from the application viewpoint. ${ }^{2}$ In terms of applications, metal cladding on $\mathrm{MgB}_{2}$ or for any superconducting wire is the most important part vis-à-vis the critical current density. A recent report has revealed that $\mathrm{Fe}$ can be a potential candidate as a practical cladding metal or as a diffusion barrier for $\mathrm{MgB}_{2}$ wire fabrication. ${ }^{2}$ Recent NMR investigations on $\mathrm{Fe}$ substituted $\mathrm{MgB}_{2}$ have revealed that the density of states close to the Fermi level remains nearly unaffected upon its substitution up to $3 \% .^{23}$ It is well known that Seebeck coefficient is very sensitive to the changes in the DOS around the Fermi level, particularly doping induced changes in the electronic structure of $\mathrm{MgB}_{2}$ can be effectively investigated. ${ }^{11,21,22}$ Therefore, a further systematic investigation on the effect of $\mathrm{Fe}$ substitution in the transport properties of $\mathrm{MgB}_{2}$ is in the right perspective. In the present work we have carried out electrical resistivity, thermal conductivity, and Seebeck coefficient measurements on $\mathrm{Mg}_{1-x} \mathrm{Fe}_{x} \mathrm{~B}_{2}$ ( $x=0 \%$ to $3 \%$ ) samples from 10 to $300 \mathrm{~K}$ to elucidate some of the above-mentioned aspects along with the theoretical analysis of the obtained data.

\section{EXPERIMENT}

Polycrystalline samples of $\mathrm{Mg}_{1-x} \mathrm{Fe}_{x} \mathrm{~B}_{2}$ with $x$ varying from 0 to $3 \%$ have been synthesized by the solid state reaction route. Synthesis details and structural characterization of these samples have already been described elsewhere. ${ }^{23}$ Nominal substitution level of $\mathrm{Fe}$ is limited up to $3 \%$, beyond which appearance of a significant amount of impurity phases is noticed. Both the lattice constants, $a$ and $c$ were found to decrease with increasing $\mathrm{Fe}$ content. The atomic radii values of $\mathrm{Fe}$ and $\mathrm{Mg}$ and the linear variation in lattice constants up to $1.2 \%$ of Fe (cf. Fig. 2 of Ref. 23) clearly indicate the successful substitution of $\mathrm{Fe}$ at $\mathrm{Mg}$ site as per the Vegard's relationship. Beyond $1.2 \% \mathrm{Fe}$, deviation from the linear variation has been noticed, which is most likely due to the solubility limit of $\mathrm{Fe}$ in the $\mathrm{MgB}_{2}$ lattice and the gradual formation of impurity phases. However, since the concentration of $\mathrm{Fe}$ is only $3.0 \%$ in the impurity phase system, from the viewpoints of electrical and thermal conductions we may formally treat the Fe sites of the impurity phase, if any, also as scatterer sites for the carriers of the host $\mathrm{MgB}_{2}$ system. In fact, according to Ref. 23, the $3.0 \%$ Fe sample is found to have almost the same density of states at the Fermi level as other samples. This implies that the possible secondary phase in the $3.0 \% \mathrm{Fe}$ sample does not affect the electronic nature of the carriers of the system. When it is so, the secondary phase (involving Fe, etc.) may be treated as a source for the scattering of the conduction electrons or lattice vibrations. More detailed clarification about the impurity phases and solubility of Fe can be seen in Ref. 23. Electrical resistivity $\rho(T)$ measurements have been carried out by the standard four-probe method. Seebeck coefficient $S(T)$ and thermal conductivity $\kappa(T)$ measurements have been performed simultaneously by a heat pulse technique in a helium closed-cycle refrigerator
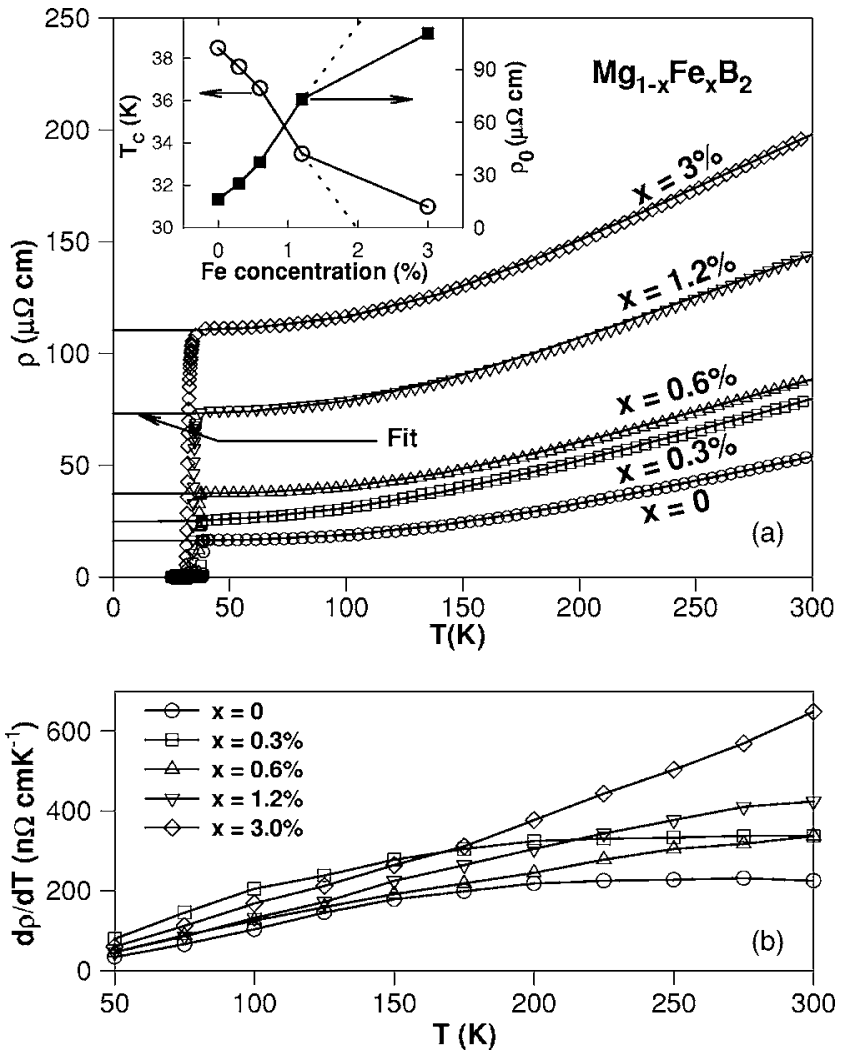

FIG. 1. (a) The temperature-dependent electrical resistivity $\rho(T)$ of $\mathrm{Mg}_{1-x} \mathrm{Fe}_{x} \mathrm{~B}_{2}$ alloys for $x=0.00-0.03$. The solid lines are fit for the experimental data with Eq. (3). Inset, superconducting transition temperature $T_{C}$ (open circles) and residual resistivity (RR) (filled squares) as a function of Fe concentration. (b) The slope $d \rho / d T$ $(50-300 \mathrm{~K})$.

from $10-300 \mathrm{~K}$. Detailed description about the experimental techniques can be found elsewhere. ${ }^{24}$

\section{RESULTS AND DISCUSSION}

\section{A. Electrical resistivity}

The temperature-dependent electrical resistivity $\rho(T)$ of the $\mathrm{Mg}_{1-x} \mathrm{Fe}_{x} \mathrm{~B}_{2}$ alloys ( $\left.x=0.00-0.03\right)$ is shown in Fig. 1(a). From these measurements we obtain the superconducting transition temperature in a way described by Osofsky et al. ${ }^{25}$ These values of $T_{C}$, presented in Table I and plotted in the inset of Fig. 1(a), are found to be consistent with the magnetization measurements. ${ }^{23}$ While the transition has been very sharp (less than $0.2 \mathrm{~K}$ ) for the pristine sample, significant transition broadening $\Delta T_{C}$ could be noticed with increasing Fe content $\left(\Delta T_{C} \sim 2 \mathrm{~K}\right.$ for $x=3 \%$ sample). From the inset of Fig. 1(a), it can be seen that the $T_{C}$ depression is rather linear up to $x=1.2 \%$ with a rate $d T_{C} / d x \sim 3 \mathrm{~K} / \%$, beyond which deviation from linearity with $x$ was observed. An important effect of this type of $T_{C}$ variation with $x$ in the $\mathrm{Fe}$ substituted samples (and also in the Al substituted samples of Ref. 11) is that the Abrikosov-Gorkov pair breaking theory of $T_{C}$ degradation, ${ }^{26}$ which requires sharper $T_{C}$ degradation with increasing $x$, may not be applicable in the 
TABLE I. Values of $T_{C}$, residual resistivity $\rho_{0}(x)$ and the parameters $\gamma_{\sigma}, \gamma_{\pi}, A, m$, and $\theta_{D}$ obtained by fitting Eq. (3) with the observed resistivity for samples of $\mathrm{Mg}_{1-x} \mathrm{Fe}_{x} \mathrm{~B}_{2}$ with various $x$.

\begin{tabular}{cccccccc}
\hline \hline$X$ & $\begin{array}{c}T_{C} \\
(\mathrm{~K})\end{array}$ & $\begin{array}{c}\rho_{0}(x) \\
(\mu \Omega \mathrm{cm})\end{array}$ & $\begin{array}{c}\gamma_{\sigma} \\
(\mathrm{meV})\end{array}$ & $\begin{array}{c}\gamma_{\pi} \\
(\mathrm{meV})\end{array}$ & $\begin{array}{c}A_{0} \\
\left(10^{7} \Omega \mathrm{s}^{-1} \mathrm{~K}^{-1}\right)\end{array}$ & $m$ & $\begin{array}{c}\theta_{D} \\
(\mathrm{~K})\end{array}$ \\
\hline 0.00 & 38.8 & 16.3 & 10.2 & 43.7 & 1.32 & 2.95 & 1076 \\
0.003 & 37.9 & 25.1 & 12.6 & 77.1 & 1.70 & 2.48 & 1043 \\
0.006 & 37.0 & 37.3 & 16 & 136.2 & 1.60 & 3.63 & 1021 \\
0.012 & 34.9 & 73.1 & 24.7 & 385.3 & 1.67 & 2.91 & 1152 \\
0.03 & 32.9 & 110.4 & 33.4 & 771.8 & 1.63 & 3.10 & 1131 \\
\hline \hline
\end{tabular}

present case. It is worth mentioning that the residual resistivity $(\mathrm{RR}) \rho_{0}$ obtained from the measurements appears to increase with increasing Fe substitution [see Table I and inset of Fig. 1(a)]. Similar to that of the $T_{C}$ variation, the dependence of RR deviates from linearity at $x=1.2 \%$. Actually the similar linear dependences of $T_{C}$ and RR is expected in standard models, since $d T_{C} / d x$ and $d \rho_{0} / d x$ are determined by the same parameter, the normal state scattering rate, for both magnetic and nonmagnetic impurities.

Resistivity in $\mathrm{MgB}_{2}$ can be considered as the outcome of the scattering processes of carriers of $\sigma$ and $\pi$ bands with defects (point defects, sheetlike faults, dislocations, etc.), impurities $(\mathrm{Fe}$, etc.) and phonons. In fact, according to Lue et $a l .{ }^{23}$ there is no magnetic moment on Fe in the $\mathrm{Mg}_{1-x} \mathrm{Fe}_{x} \mathrm{~B}_{2}$ samples. Thus we do not expect the presence of any electronspin scattering process in the system. The resistivity $\rho_{b}$ due to the combined scattering of the carriers of the $\sigma$ and $\pi$ bands with defects, impurities, and phonons is given by

$$
\rho_{b}(T)=\rho_{\mathrm{def}, b}+\rho_{\mathrm{imp}, b}+\rho_{\mathrm{ph}, b}(T) .
$$

Here $\rho_{\mathrm{def}, b}$ and $\rho_{\mathrm{imp}, b}$ are temperature-independent resistivities due to the scattering of $b$-band electrons by defects and impurities, respectively. The quantity $\rho_{\mathrm{ph}, b}$ in Eq. (1) is the resistivity due to scattering of $b$-band electrons by phonons. $\rho_{\mathrm{ph}, b}$ is temperature dependent and is, in general, given by the Bloch-Gruneisen expression $^{27}$

$$
\rho_{\mathrm{ph}, b}=A_{b}(m-1) \theta_{D}\left(T / \theta_{D}\right)^{m} \int_{0}^{\theta_{D} / T} \frac{z^{m}}{\left(e^{z}-1\right)\left(1-e^{-z}\right)} d z .
$$

Here $A_{b}$ depends on the band parameters via $A_{b}=\frac{A_{0} \nu_{F, b}}{\omega_{p, b}^{2}} . A_{0}$ is independent of band specifications, and of $m$ and $\theta_{D}^{p, b}$ (Debye temperature). $\nu_{F, b}$ is the Fermi velocity and $\omega_{p, b}$ is the plasma frequency both corresponding to the band $b$. We have obtained this dependence of $A_{b}$ on $\nu_{F, b}$ and $\omega_{p, b}$ by considering Eq. (7.63) of Ziman. ${ }^{28}$ According to Ref. 29 the values of $\nu_{F, b}$ and $\omega_{p, b}$ for $\mathrm{MgB}_{2}$ are $\nu_{F, \sigma}=2.4 \times 10^{5} \mathrm{~m} / \mathrm{s}, \nu_{F, \pi}$ $=5.63 \times 10^{5} \mathrm{~m} / \mathrm{s}, \quad \omega_{p, \sigma}=2.27 \mathrm{eV}$, and $\omega_{p, \pi}=6.19 \mathrm{eV}$. The overall resistivity $\rho(T)$ of the system due to the contribution of the $\sigma$ and $\pi$ bands is given by ${ }^{30}$

$$
\frac{1}{\rho(T)}=\frac{1}{\rho_{\sigma}(T)}+\frac{1}{\rho_{\pi}(T)} .
$$

From this equation the residual resistivity is given by

$$
\rho_{0}=\frac{\rho_{0, \sigma} \rho_{0, \pi}}{\left(\rho_{0, \sigma}+\rho_{0, \pi}\right)}
$$

where $^{24}$

$$
\rho_{0, b}=\frac{\gamma_{b}}{\varepsilon_{0} \omega_{p b}^{2}} .
$$

Here $\gamma_{b}$ is the sum of the scattering rates of the $b$-band carriers with the impurity and defects. $\varepsilon_{0}=8.85$ $\times 10^{-12} \mathrm{~F} / \mathrm{m}$ is the free space permittivity. Using the above expressions along with the values of $\nu_{F, b}$ and $\omega_{p, b}$ we have fitted the experimental data with Eq. (3) in terms of the parameters $\gamma_{\sigma}, \gamma_{\pi}, A_{0}, m$, and $\theta_{D}$ for various samples of $\mathrm{Mg}_{1-x} \mathrm{Fe}_{x} \mathrm{~B}_{2}$. The values of these fitting parameters are tabulated in Table I. An excellent fitting of Eq. (3) to the experimental data has been achieved with these parameters.

From Table I we see that $\gamma_{\sigma}<\gamma_{\pi}$ for all the considered samples. This is what we expect from the work of Mazin et $a l .{ }^{30}$ From the values of $\gamma_{\sigma}$ and $\gamma_{\pi}$ for $x=0.0$ we estimate the contributions of the $\sigma$ and $\pi$ carriers to the residual resistivity. We obtain, $\rho_{\text {def }, \sigma}=44.3 \mu \Omega \mathrm{cm}$ and $\rho_{\text {def }, \pi}=25.6 \mu \Omega \mathrm{cm}$. For $x>0, \rho_{0, b}$ is the direct sum of the values of the resistivities due to defects $\left(\rho_{\text {def, } b}\right)$ and impurities $\left(\rho_{\text {imp }, b}\right)$. That is to say

$$
\rho_{0, b}=\rho_{\mathrm{def}, b}+\rho_{\mathrm{imp}, b} .
$$

Another information that we can draw on the basis of Table I is that the temperature dependence of $\rho(T)$, as signified by the values of $m$ and $\theta_{D}$, varies nonmonotonically with increasing $x$. This is because the uncertainties in the fitting of the values of $m$ and $\theta_{D}$ are much less than the difference in the values of $m$ or $\theta_{D}$ for different $x$. For example, $100[m(x=0.0)-m(x=0.003)] / m(x=0.0)=15.9 \%$ is much larger than the $2.0 \%$ uncertainty in the fitting of $m$. It may be noted here that the uncertainty in the fitting of the $\theta_{D}$ values is less than $0.5 \%$. Since the values of $\theta_{D}$ vary by less than $12 \%$ for the considered values of $x$, and since the highest temperature for which measurements are done $(300 \mathrm{~K})$ is much less than $\theta_{D}(x)$, we do not expect a significant effect of $\theta_{D}$ on the temperature variation of $\rho(T)$. Then the sensitivity of the temperature dependence $\rho(T)$ is governed mainly by the parameter $m$ [cf. Eq. (2)]. From Table I we see that the value of $m$ decreases by about $16 \%$ for the $0.3 \%$ sample. But with a $0.6 \%$ substitution of $\mathrm{Fe}$ the value of $m$ increases sharply to 3.63 and then decreases for $1.2 \%$ sample. For the 


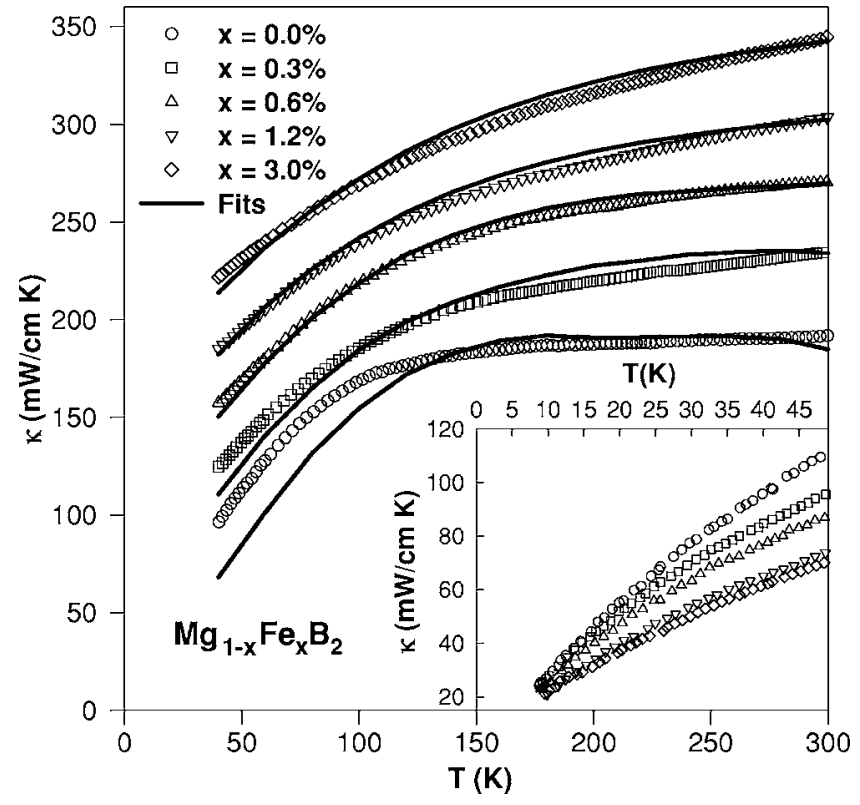

FIG. 2. The temperature-dependent thermal conductivity for $\mathrm{Mg}_{1-x} \mathrm{Fe}_{x} \mathrm{~B}_{2}$ alloys $(x=0.00-0.03)$. The solid lines are fit for the experimental data with Eq. (7). The inset $(10-50 \mathrm{~K})$ is to clarify that there is no hump in $\kappa$ below $T_{C} \sim 39 \mathrm{~K}$. The data is shifted by various amounts for clear vision.

$3.0 \%$ sample the value of $m$ is equal to that for the unsubstituted sample within 5\%. Reported results of Lorenz et al. ${ }^{11}$ on Al-substituted samples of $\mathrm{MgB}_{2}$ are also expected to lead to such nonmonotonic variation of $m$ as obtained here.

In order to gauge such a nonmonotonic variation of $m$ with the Fe content we consider the $m$ dependence of $\rho(T)$. Following Eqs. (2) and (3) we obtain the following form of the slope $d \rho / d T$ as $s=(d \rho / d T)=\left(T / \theta_{D}\right)^{m-1} m^{m+2}(T \rightarrow 0)$. The first term $\left(T / \theta_{D}\right)^{m-1}$ decreases sharply with $m$ as $\left(T / \theta_{D}\right) \ll 1$ for $T \rightarrow 0$, in contrast to the second factor $m^{m+2}$ which increases with $m$. However, the variation of $m^{m+2}$ is relatively much slower. For example, taking $T=10 \mathrm{~K}, \theta_{D}=10^{3} \mathrm{~K}$, $\left(T / \theta_{D}\right)^{m-1}$ decreases by a factor of $10^{4}$ while the factor $m^{m+2}$ increases only by 256 for $m$ increasing from 2 to 4 . This implies that for low $T(T \rightarrow 0)$ the variation of the slope $s$ is dominated by the factor $\left(T / \theta_{D}\right)^{m-1}$. Since $\left(T / \theta_{D}\right)^{m-1}$ decreases with increasing $m, s$ will also decrease with $m$. In Fig. 1(b) it is the slope of the $x=0.3 \%$ Fe sample that is significantly different near $T=50 \mathrm{~K}$. So, we expect that at least this sample will follow the same higher value of $(d \rho / d T)$ for low $T(T \rightarrow 0)$ also. The value of $s$ is therefore, expected to be the highest for the $x=0.3 \%$ Fe sample. In view of the above-mentioned variation of $s$ with $m$ we may say that the $x=0.3 \% \mathrm{Fe}$ sample will correspond to the lowest value of $m$ (cf. Table I).

\section{B. Thermal conductivity}

The temperature-dependent thermal conductivity $\kappa(T)$ data of the various Fe-substituted $\mathrm{MgB}_{2}$ samples are presented in Fig. 2. The absence of any hump in $\kappa$ below $T_{C}$ (inset of Fig. 2) agrees well with the reported $\kappa(T)$ data. ${ }^{21,27}$
It would be appropriate to mention here that the data in Fig. 2 has been shifted by different amounts for the sake of clear qualitative depiction. For $x=0$, the thermal conductivity $\kappa(T)$ shows a shoulder near $110 \mathrm{~K}$. Other groups have also observed a shoulder in $\kappa$ for the pristine sample..$^{21,27}$ With increasing $x$ the shoulder near $T \sim 110 \mathrm{~K}$ becomes weaker and eventually fades away for the $3.0 \%$ sample. This is in contrast to the appearance of a shoulder for all $x$ in $\mathrm{Cr}$ substituted $\mathrm{MgB}_{2} \cdot{ }^{21}$ In the Fe-substituted samples, the thermal conductivity $\kappa$ decreases with $x$ for $T \leq 260 \mathrm{~K}$. At $300 \mathrm{~K}, \kappa$ is highest for the $0.3 \%$ sample and lowest for the $1.2 \%$ sample. As far as the quantitative values of $\kappa$ are concerned it may be noted that the room temperature $\kappa$ value $(\sim 190 \mathrm{~mW} / \mathrm{cm} \mathrm{K})$ is nearly invariant of the $\mathrm{Fe}$ content.

We may write the total thermal conductivity of $\mathrm{Mg}_{1-x} \mathrm{Fe}_{x} \mathrm{~B}_{2}$ as composed of two parts-the first $\left(\kappa_{\mathrm{el}}\right)$ is the electronic contribution due to conduction electrons and the second $\left(\kappa_{\mathrm{ph}}\right)$ is the lattice contribution due to the phonons. That is to say,

$$
\kappa=\kappa_{\mathrm{el}}+\kappa_{\mathrm{ph}} .
$$

The electronic contribution $\kappa_{\mathrm{el}}$ may be expressed in terms of the dc resistivity $\rho(T)$, Eq. (3), by using the WiedemannFranz law ${ }^{31}$

$$
\kappa_{\mathrm{el}}=\frac{L_{0} T}{\rho(T)} .
$$

$L_{0}$ being the Lorenz number.

From Eqs. (7) and (8) we have found that at any given temperature the lattice contribution to the experimental thermal conductivity follows the relation

$$
\kappa_{\mathrm{ph}}\left(x^{\prime}, T\right)<\kappa_{\mathrm{ph}}\left(x^{\prime \prime}, T\right) \text { for } x^{\prime}<x^{\prime \prime} .
$$

Here $x^{\prime}, x^{\prime \prime}=0.0,0.003,0.006,0.012$, and 0.03 .

Since $\rho(T)$ involves contributions from both $\sigma$ and $\pi$ bands of $\mathrm{MgB}_{2}, \kappa_{\mathrm{el}}$ also involves contributions from these two bands. The phonon contribution $\kappa_{\mathrm{ph}}$, however, has nothing to do with the electronic bands $\sigma$ and $\pi$ and is entirely contributed by the lattice. In general, we may express the lattice thermal conductivity $\kappa_{\mathrm{ph}}$ by ${ }^{32}$

$$
\kappa_{\mathrm{ph}}(T)=t^{3} \int_{0}^{\theta_{D} / T} \frac{z^{4}}{\left(e^{z}-1\right)\left(1-e^{-z}\right) K(t, z)} d z .
$$

Here $t=T / T_{C}$ is reduced transition temperature, and $K(t, z)$ is the sum of the scattering rates of phonons due to different scattering sources. From the inset of Fig. 2 we see that there is no hump in $\kappa$ below $T_{C}$. Thus, according to Ref. 32 we do not consider the scattering of phonons by electrons in $K(t, z)$. There are then four main sources of phonon scattering events which we feel are important in deciding the values of $\kappa_{\mathrm{ph}}$ in $\mathrm{MgB}_{2}$. They are (1) grain boundaries in the sample, (2) point defects, (3) strain fields of sheetlike faults, and (4) strain fields of dislocations. We thus write ${ }^{32}$

$$
K(t, z)=K_{0}+K_{\mathrm{pd}} t^{4} z^{4}+K_{\mathrm{sf}} t^{2} z^{2}+K_{\mathrm{dis}} t z .
$$

Here $K_{0}$ is the boundary scattering rate, $K_{\mathrm{pd}}$ is the scattering rate of phonons from the point defects, $K_{\mathrm{sf}}$ is the scattering 

(10).

TABLE II. Values of the parameters $K_{0}, K_{\mathrm{pd}}, K_{\mathrm{sf}}$, and $K_{\text {dis }}$ of Eq.

\begin{tabular}{ccccc}
\hline \hline & $\begin{array}{c}K_{0} \\
(\mathrm{~cm} \mathrm{~K} / \mathrm{mW})\end{array}$ & $\begin{array}{c}K_{\mathrm{pd}} \\
(\mathrm{cm} \mathrm{K} / \mathrm{mW})\end{array}$ & $\begin{array}{c}K_{\text {sf }} \\
(\mathrm{cm} \mathrm{K} / \mathrm{mW})\end{array}$ & $\begin{array}{c}K_{\text {dis }} \\
(\mathrm{cm} \mathrm{K} / \mathrm{mW})\end{array}$ \\
\hline 0.00 & 3.55 & 0.35 & 319 & 28.4 \\
0.003 & 0.94 & 1.22 & 85 & 7.6 \\
0.006 & 0.63 & 2.26 & 57 & 5.0 \\
0.012 & 0.64 & 1.40 & 57 & 5.1 \\
0.03 & 0.79 & 0.79 & 71 & 7.9 \\
\hline \hline
\end{tabular}

rate from sheetlike faults, and $K_{\text {dis }}$ is the scattering rate from dislocations.

In order to maintain a consistency of our analysis we have used those values of $\theta_{D}$ in Eq. (10) which we have obtained from $\rho$ analysis (Table I). The fitting parameters $K_{0}, K_{\mathrm{pd}}, K_{\mathrm{sf}}$, and $K_{\text {dis }}$ are given in Table II. These parameters do not follow a monotonic variation with $x$. The main reason for this is that the residual resistivity $\rho_{0}$ is not linear in $x$, and that the temperature dependence of $\rho(T)$ is also nonmonotonic (cf. values of $m$ in Table I). Despite this we find from Table II that the parameters $K_{0}$ and $K_{\mathrm{pd}}$ vary with $x$ in a manner to oppose each others' effect. Then, for $x \leq 0.012$, the decrease of $K_{\text {sf }}$ and $K_{\text {dis }}$ with increasing $x$ may be understood as a process for the relation of Eq. (9). In view of Eq. (9) the overall coupling of the phonons with defects and/or Fe decreases with increasing $x$. From the viewpoint of Table II, up to $x \approx 0.012$ the shoulder appears due to the dominance of the reduction of the effects of the boundary scattering $\left(K_{0}\right)$ and dislocations $\left(K_{\mathrm{dis}}\right)$ over the effect of the point defects $\left(K_{\mathrm{pd}}\right)$. For the $3.0 \%$ Fe content, on the other hand, the shoulder appears due to the dominance of the effect of the point defects over the defects.

Although, the parameters of Table II do not show a systematic variation with $x$, according to Eq. (10) their combined effect, or equivalently the combined effect of defects and $\mathrm{Fe}$, becomes weaker for phonons with increasing $x$. This situation is different from that of the interaction of phonons with electrons as evidenced from the variations of parameters $A_{0}$ and $m$ in Table I. The variations of $A_{0}$ with $x$ means that the electron-phonon interaction is strongest for the $x=0.003$ sample, decreasing a little with further substitution.

\section{Seebeck coefficient}

The temperature-dependent Seebeck coefficient $S(T)$ of the $\mathrm{Mg}_{1-x} \mathrm{Fe}_{x} \mathrm{~B}_{2}$ alloys with various $x$ is shown in Fig. 3. Each curve is offset by $1 \mu \mathrm{V} / \mathrm{K}$ for clarity. For the presently investigated $\mathrm{Mg}_{1-x} \mathrm{Fe}_{x} \mathrm{~B}_{2}$ samples, the common features observed in Seebeck coefficient, such as the positive sign, the small magnitude at $300 \mathrm{~K}$, the linear variation between $T_{C}$ to $\sim 150 \mathrm{~K}$ (solid lines and arrows in the figure), and the tendency to saturate at higher temperatures are in well accordance with the behavior widely reported for $\mathrm{MgB}_{2} \cdot{ }^{11,21,22,33,34}$ The transition temperatures determined from the Seebeck coefficient measurements are generally consistent with the electrical resistivity measurements for

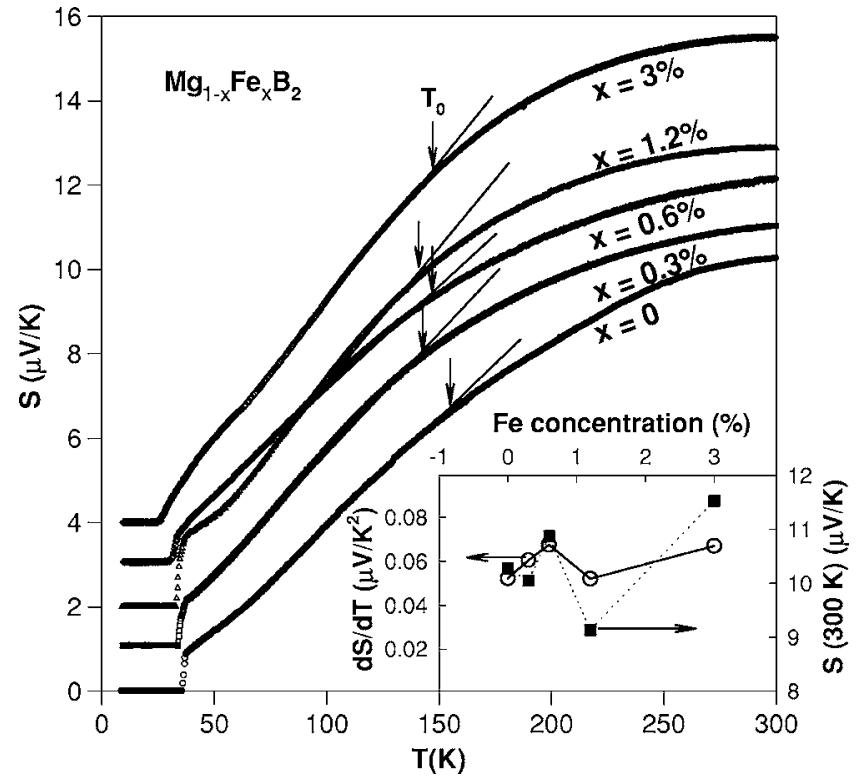

FIG. 3. The temperature-dependent Seebeck coefficient curves for $\mathrm{Mg}_{1-x} \mathrm{Fe}_{x} \mathrm{~B}_{2}$ alloys $(x=0.00-0.03)$. Inset, the slopes of Seebeck coefficient $d S / d T$ (open circles) in the linear region and the values of room-temperature Seebeck coefficient $S(300 \mathrm{~K})$ (filled squares) as a function of $\mathrm{Fe}$ concentration. No systematic variations in $d S / d T$ and $S(300 \mathrm{~K})$ with respect to Fe concentration could be noticed.

these $\mathrm{Mg}_{1-x} \mathrm{Fe}_{x} \mathrm{~B}_{2}$ alloys. The notable observation of the present results of Seebeck coefficient measurements is that the variations in $S(T)$ in the normal state are nearly composition independent, which is significantly in contrast to the general behavior observed by other substituents in $\mathrm{MgB}_{2} \cdot{ }^{11,21,22,34} \mathrm{Fe}$ is a trivalent element and effectively an electron dopant. Besides, one can expect a slight band broadening due to the increase in the lattice compression as the lattice parameters of $\mathrm{Mg}_{1-x} \mathrm{Fe}_{x} \mathrm{~B}_{2}$ alloys decrease monotonically with the $\mathrm{Fe}$ concentration. Electron doping and the band broadening generally lead to a systematic decrease in DOS around $E_{F}$ in $\mathrm{MgB}_{2}$. Further, recently reported Seebeck coefficient measurements on $\mathrm{Al}$ substituted $\mathrm{MgB}_{2}$ have correlated the changes in $E_{F}$ to the observed systematic variations in the slope of $S(T)$ between $T_{C}$ to $200 \mathrm{~K}$, ${ }^{11}$ where the value of $E_{F}$ is related to the slope of temperature-dependent Seebeck coefficient through the classical formula

$$
\frac{S}{T}=\frac{\pi^{2} k_{B}^{2}}{3 e E_{F}},
$$

assuming a one-band model with an energy-independent relaxation time. Some of the other transition elements like Co and $\mathrm{Cr}$ have shown significantly large and systematic variations in the magnitude of normal-state Seebeck coefficient upon their substitution and such variations have been attributed to the reduction in DOS. ${ }^{21,22}$ As a nonmagnetic trivalent element, one would expect that Fe should show a similar effect as Al substitution, which in turn must reduce the DOS in holelike $\sigma$-band by electron doping and the electronic topological transition of the Fermi surface from two to three 
dimensional. ${ }^{35}$ Such changes in the electronic structure can change the magnitude and as well the sign of $S$ dramatically. However, as shown in the inset of Fig. 3, no systematic correlation between the magnitude of $S(300 \mathrm{~K})$ and $d S / d T$ with respect to Fe concentration could be noticed. This is in good agreement with the earlier theoretical prediction that the DOS remains unaffected for small concentrations of dopants, and rapid reduction of DOS occurs when $T_{C}$ decreases below $\sim 25 \mathrm{~K}$ in $\mathrm{MgB}_{2}{ }^{19}$ It is worth mentioning that previous NMR investigation on $\mathrm{Fe}$ substituted $\mathrm{MgB}_{2}$ samples revealed a nearly unaffected DOS and the observed variations in $T_{C}$ has been attributed to the decrease in phonon frequency and/or reduced electron-phonon coupling strength as a result of disorder. ${ }^{23}$ The present Seebeck coefficient measurements also indicate no significant changes in DOS for Fe substitution levels up to $3 \%$, which is in strong support to the NMR investigations.

In a polycrystalline sample, similar to Hall effect, the measured $S$ is the net value of the two bands, $\sigma$ and $\pi{ }^{36,37}$ Out of $\sigma$ and $\pi$ bands, from the positive value of $S$ it is clear that the holelike $\sigma$-band dominates the thermoelectric transport in these $\mathrm{Mg}_{1-x} \mathrm{Fe}_{x} \mathrm{~B}_{2}$ alloys. To maintain a nearly unchanged DOS we require a compensating effect and/or charge transferring between $\sigma$ and $\pi$ bands upon Fe substitution. The observed random variations in the roomtemperature Seebeck coefficient as well as the slope in the present study clearly reveal that more than one mechanism may be competing with each other in altering the DOS.

\section{Discussion}

Among the dopants, Mn shows rapid reduction in $T_{C}$ for a given concentration and as expected, as a magnetic element, Mn can have dramatic effect on $T_{C}$ due to the pair breaking effect through spin-flip scattering. ${ }^{13,14,17}$ Although, Fe substitution shows weaker decrease in $T_{C}$ as compared with Mn, the decrease in $T_{C}$ is more pronounced than the other dopants like Al, Sc, C, etc., where the depression of $T_{C}$ has been attributed to the reduction of DOS through the band filling effect. ${ }^{7} 10,12,19-22$ The previous theoretical study has predicted the nonmagnetic nature of $\mathrm{Fe}$ in $\mathrm{MgB}_{2}$ lattice and subsequently NMR investigation has confirmed the entirely nonmagnetic nature of $\mathrm{Fe}$ and as well as the absence of any traceable magnetic impurities or impurity phases (both can act as a strong source for spin-flip scattering), and nearly unchanged DOS. ${ }^{17,23}$ The extrinsic disorder, disorder induced by neutron irradiation shows significant variations in $T_{C}$, nevertheless decrease in DOS has also been observed. ${ }^{38,39}$ On the other hand, according to Putti and Chen et al. ${ }^{27,40}$ in undoped samples, the superconducting transition temperature is insensitive to the intrinsic disorder to a considerable extent, whereas it affects the normal state transport properties significantly. It is interesting to note that only negligible variations in $T_{C}(\sim 1 \mathrm{~K})$ have been reported for changes in residual resistivity ratio (RRR) from 3 to 8 , which is much larger when compared with the RRR variations noticed among the present Fe concentrations $(\sim 2-4)$. It is common to note that the presence of $\mathrm{MgO}$ impurity phases, which can be one of the major sources of disorder and may lead to increase in RRR, nevertheless have only negligible effect on $T_{C}$.

Upon substitution of other elements, except $\mathrm{Mn}$, the $T_{C}$ shows much smaller variations for dopant concentrations equivalent to the present $\mathrm{Fe}$ concentration ranges. ${ }^{7,11,20-22,34,41}$ Particularly, the $T_{C}$ depression rate is more pronounced than the other well-known substituent such as $\mathrm{Al}$ with the similar variations in the lattice constant upon its substitution. As mentioned earlier, $\mathrm{Al}$ and $\mathrm{Fe}$ are trivalent, nonmagnetic in $\mathrm{MgB}_{2}$ lattice, and lattice constant decreases upon their substitution. The induced disorder as a result of their substitution at $\mathrm{Mg}$ lattice (substitutional disorder), may not have any dramatic difference in the extent of disorder between them and hence the substitutional disorder should show nearly identical effect on the superconducting transition temperatures. Further, no traceable magnetic impurities were noticed by NMR measurements, ${ }^{23}$ and hence the effect of small amounts of $\mathrm{FeB}$ impurity phase for higher Fe contents can be treated similar to the inevitable $\mathrm{MgO}$ impurity phase, which is also nonmagnetic and can be one of the sources of disorder. As mentioned earlier, the superconducting transition temperature is insensitive to disorder to a considerable extent. Therefore, in the present case, the substitutional disorder (which we treat as intrinsic) as a result of $\mathrm{Fe}$ substitution, if any, may affect only the normal state properties rather than the superconducting properties.

These findings, nonmagnetic nature of $\mathrm{Fe}$ in $\mathrm{MgB}_{2}$, modest $T_{C}$ depression rate when compared with $\mathrm{Mn}$, much pronounced $T_{C}$ depression rate when compared with other elements like $\mathrm{Al}$, and nearly unaffected $\mathrm{DOS}$ place the $\mathrm{Fe}$ substitution in a very unique situation and bring up the importance of the phonon contributions to the variation of $T_{C}$ for $\mathrm{MgB}_{2}$. For a BCS phonon-mediated superconductor, it is well known that the $T_{C}$ is proportional to the phonon frequency $\omega$, following the McMillian formula. The unusual high critical temperature for $\mathrm{MgB}_{2}$ is partially attributed to the high frequency of the $E_{2 g}$ phonon mode $(\omega$ $=64-82 \mathrm{meV}){ }^{4,19}$ The present Fe substitution is expected to specifically modify the phonon spectrum, in order to alter the phonon frequency and/or electron-phonon coupling strength. However, absence of such an effect in the isoelectronic (like $\mathrm{Al}$ ) and other dopants is an open question. Most probably, such a scenario depends on the specific nature of the given substituent element.

\section{CONCLUSIONS}

We have investigated the superconducting and electronic properties of $\mathrm{Fe}$ substituted $\mathrm{Mg}_{1-x} \mathrm{Fe}_{x} \mathrm{~B}_{2}$ samples with $x$ $=0 \%, 0.3 \%, 0.6 \%, 1.2 \%$, and $3 \%$ superconductor by electrical resistivity, thermal conductivity, and Seebeck coefficient measurements from $10-300 \mathrm{~K}$. Superconducting transition temperature $\left(T_{C}\right)$ decreases rather linearly as a function of $\mathrm{Fe}$ concentration up to $x=1.2 \%$, beyond which the solubility limit of Fe in the $\mathrm{MgB}_{2}$ lattice was noticed. The two-band model provides an excellent description of the resistivity data in terms of the Bloch-Grueneisen model and the coupling of the carriers with defects and/or impurity increases with $\mathrm{Fe}$ substitution nonmonotonically between $22 \%$ and 
$30 \%$. Thermal conductivity of the pristine material is seen to be the highest and exhibits a shoulder near $110 \mathrm{~K}$ which gradually fades out with increasing $x$. The overall coupling of phonons with defects and/or impurities as signified by the lattice thermal conductivity decreases with increasing $\mathrm{Fe}$ content. It has been found that the room-temperature Seebeck coefficient as well as the slope of Seebeck coefficient in the linear region (from $T_{C}$ to about $150 \mathrm{~K}$ ) show little change with respect to the Fe substitution, indicating that the DOS near the Fermi level remains nearly unaffected in these $\mathrm{Mg}_{1-x} \mathrm{Fe}_{x} \mathrm{~B}_{2}$ alloys, consistent with the previous NMR report. ${ }^{23}$ The conclusions from the present results on the $\mathrm{Fe}$ substituted $\mathrm{Mg}_{1-x} \mathrm{Fe}_{x} \mathrm{~B}_{2}$ alloys, a modest $T_{C}$ depression rate when compared with $\mathrm{Mn}$, a much pronounced $T_{C}$ depression rate when compared with other elements like $\mathrm{Al}$, and nearly unaffected DOS rules out the possibility of spin-flip scattering and/or reduction of the DOS as well as disorder as a cause for the observed variations in $T_{C}$ and place the role of
Fe in the $\mathrm{MgB}_{2}$ lattice as a distinct one. The absence of consistent variation in the electronic and superconducting properties between Fe and other similar substituent elements (with parallel characteristics like the nonmagnetic and trivalent element $\mathrm{Al}$ ) substituted $\mathrm{MgB}_{2}$ suggest that the specific electronic nature of the given substituent element $(\mathrm{Fe})$ plays an important role in altering the phonon frequency and/or electron-phonon coupling strength.

\section{ACKNOWLEDGMENTS}

The authors are thankful to S. K. Joshi, NPL, New Delhi and M. A. H. Ahsan, Department of Physics, JMI, New Delhi for valuable discussions. One of the authors (B.G.) is grateful to CSIR for financial help under Contract No. CSIR-

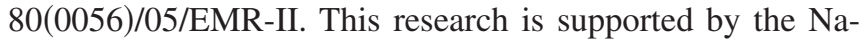
tional Science Council of Taiwan under Contract No. NSC94-2112-M-259-012 (Y.K.K.).
*Electronic address: ykkuo@mail.ndhu.edu.tw

${ }^{1}$ J. Nagamastu, N. Nakagawa, T. Muranaka, Y. Zenitani, and J. Akimitsu, Nature (London) 410, 63 (2001).

${ }^{2}$ S. Jin, H. Mavoori, C. Bower, and R. B. van Dover, Nature (London) 411, 563 (2001).

${ }^{3}$ I. I. Mazin and V. P. Antropov, Physica C 385, 49 (2003), cf. Fig. 2.

${ }^{4}$ J. M. An and W. E. Pickett, Phys. Rev. Lett. 86, 4366 (2001).

${ }^{5}$ J. M. Rowell, Supercond. Sci. Technol. 16, R17 (2003).

${ }^{6}$ C. H. Cheng, Y. Zhao, X. T. Zhu, J. Nowotny, C. C. Sorrell, T. Finlayson, and H. Zhang, Physica C 386, 588 (2003).

${ }^{7}$ M. Kühberger and G. Gritzner, Physica C 370, 39 (2002).

${ }^{8}$ Y. Moritomo and Sh. Xu, arXiv:cond-mat/0104568 (unpublished).

${ }^{9}$ D. Di Castro, M. Ortolani, E. Cappelluti, U. Schade, N. D. Zhigadlo, and J. Karpinski, Phys. Rev. B 73, 174509 (2006).

${ }^{10}$ S. Agrestini, C. Metallo, M. Filippi, G. Campi, C. Sanipoli, S. De Negri, M. Giovannini, A. Saccone, A. Latini, and A. Bianconi, J. Phys. Chem. Solids 65, 1479 (2004).

${ }^{11}$ B. Lorenz, R. L. Meng, Y. Y. Xue, and C. W. Chu, Phys. Rev. B 64, 052513 (2001).

${ }^{12}$ O. de la Peña, A. Aguayo, and R. de Coss, Phys. Rev. B 66, 012511 (2002).

${ }^{13}$ K. Rogacki, B. Batlogg, J. Karpinski, N. D. Zhigadlo, G. Schuck, S. M. Kazakov, P. Wagli, R. Puzniak, A. Wisniewski, F. Carbone, A. Brinkman, and D. van der Marel, Phys. Rev. B 73, 174520 (2006).

${ }^{14}$ R. S. Gonnelli, D. Daghero, G. A. Ummarino, A. Calzolari, M. Tortello, V. A. Stepanov, N. D. Zhigadlo, K. Rogacki, J. Karpinski, F. Bernardini, and S. Massidda, Phys. Rev. Lett. 97, 037001 (2006).

${ }^{15}$ Y. D. Gao, J. Ding, G. V. S. Rao, and B. V. R. Chowdari, J. Appl. Phys. 93, 8656 (2003).

${ }^{16}$ S. X. Dou, S. Soltanian, Y. Zhao, E. Getin, Z. Chen, O. Shcherbakova, and J. Horvat, Supercond. Sci. Technol. 18, 710 (2005).

${ }^{17}$ P. J. T. Joseph and P. P. Singh, Solid State Commun. 141, 390 (2007).
${ }^{18}$ R. J. Cava, H. W. Zandbergen, and K. Inumaru, Physica C 385, 8 (2003), and references therein.

${ }^{19}$ J. Kortus, O. V. Dolgov, R. K. Kremer, and A. A. Golubov, Phys. Rev. Lett. 94, 027002 (2005), and references therein.

${ }^{20}$ A. Tampieri, G. Celotti, S. Sprio, D. Rinaldi, G. Barucca, and R. Caciuffo, Solid State Commun. 121, 497 (2002).

${ }^{21}$ M. E. Yakinci, Y. Balci, and M. A. Aksan, Physica C 408-410, 684 (2004).

${ }^{22}$ M. A. Aksan, M. E. Yakinci, and A. Güldeste, J. Alloys Compd. 424, 33 (2006).

${ }^{23}$ C. S. Lue, T. H. Su, B. X. Xie, S. K. Chen, J. L. MacManusDriscoll, Y. K. Kuo, and H. D. Yang, Phys. Rev. B 73, 214505 (2006).

${ }^{24}$ C. S. Lue, Y.-K. Kuo, S.-N. Hong, S. Y. Peng, and C. Cheng, Phys. Rev. B 71, 064202 (2005).

${ }^{25}$ M. S. Osofsky, R. J. Soulen, Jr., S. A. Wolf, J. M. Broto, H. Rakoto, J. C. Ousset, G. Coffe, S. Askenazy, P. Pari, I. Bozovic, J. N. Eckstein, and G. F. Virshup, Phys. Rev. Lett. 71, 2315 (1993).

${ }^{26}$ A. A. Abrikosov and L. P. Gorkov, Sov. Phys. JETP 12, 1243 (1961).

${ }^{27}$ M. Putti, V. Braccini, E. Galleani, F. Napoli, I. Pallecchi, A. S. Siri, P. Manfrinetti, and A. Palenzona, Supercond. Sci. Technol. 16, 188 (2003).

${ }^{28}$ J. M. Ziman, Principle of the Theory of Solids (Cambridge University Press, Cambridge, 1972), pp. 224 and 225.

${ }^{29}$ A. Brinkman, A. A. Golubov, H. Rogalla, O. V. Dolgov, J. Kortus, Y. Kong, O. Jepsen, and O. K. Andersen, Phys. Rev. B 65, 180517(R) (2002).

${ }^{30}$ I. I. Mazin, O. K. Andersen, O. Jepsen, O. V. Dolgov, J. Kortus, A. A. Golubov, A. B. Kuz'menko, and D. van der Marel, Phys. Rev. Lett. 89, 107002 (2002).

${ }^{31}$ E. Bauer, Ch Paul, St Berger, S. Majumdar, H. Michor, M. Giovannini, A. Saccone, and A. Bianconi, J. Phys.: Condens. Matter 13, L487 (2001).

${ }^{32}$ L. Tewordt and Th. Wölkhausen, Solid State Commun. 70, 839 (1989). 
${ }^{33}$ T. Muranaka, J. Akimitsu, and M. Sera, Phys. Rev. B 64, 020505(R) (2001).

${ }^{34}$ J. S. Ahn, E. S. Choi, W. Kang, D. J. Singh, M. Han, and E. J. Choi, Phys. Rev. B 65, 214534 (2002).

${ }^{35}$ S. Agrestini, C. Metallo, M. Filippi, L. Simonelli, G. Campi, C. Sanipoli, E. Liarokapis, S. De Negri, M. Giovanni, A. Saccone, A. Latini, and A. Bianconi, Phys. Rev. B 70, 134514 (2004).

${ }^{36}$ T. Masui and S. Tajima, Physica C 385, 91 (2003).

${ }^{37}$ A. Poddar, B. Bandyopadhyay, P. Mandal, D. Bhattacharya, P. Choudhury, U. Sinha, and B. Ghosh, Physica C 390, 191 (2003).
${ }^{38}$ R. H. T. Wilke, S. L. Bud'ko, P. C. Canfield, J. Farmer, and S. T. Hannahs, arXiv:cond-mat/0507185, Phys. Rev. B (to be published).

${ }^{39}$ A. P. Gerashenko, K. N. Mikhalev, S. V. Verkhovskii, A. E. Karkin, and B. N. Goshchitskii, Phys. Rev. B 65, 132506 (2002).

${ }^{40}$ X. H. Chen, Y. S. Wang, Y. Y. Xue, R. L. Meng, Y. Q. Wang, and C. W. Chu, Phys. Rev. B 65, 024502 (2001).

${ }^{41}$ Kiran Singh, Rajneesh Mohan, Nupinder Kaur, N. K. Gaur, Manglesh Dixit, Vilas Shelke, and R. K. Singh, Physica C 450, 124 (2006). 